\title{
détermination d'un coefficient de sécurité dans les exploitations par chambres et petits piliers abandonnés
}

\author{
par \\ J. Bonvallet \\ Ingénieur au Centre d'Études et de Recherches des charbonnages de France* \\ C. Chambon \\ Professeur à l'École des Mines de Nancy
}

II existe dans plusieurs régions françaises d'anciennes exploitations souterraines de calcaire, de craie ou de gypse, autrefois activement exploitées pour la construction et qui posent aujourd'hui des problèmes de stabilité, notamment au voisinage des centres urbains. Dans le seul département du Nord par exemple, on estime à environ 2000 ha l'étendue de l'ensemble des carrières souterraines dont certaines dateraient du VII ${ }^{\circ}$ siècle.

Pour faire face à ce problème, un Service Départemental d'Inspection des Carrières Souterraines (S. D. I.C.S.) a été créé en 1967, par le Conseil Général du Nord et placé sous l'autorité de l'arrondissement minéralogique du Nord-Pas-de-Calais, actuellement Direction Interdépartementale de l'Industrie. II a pour missions la localisation, le relevé des plans et la surveillance des carrières souterraines, ainsi que la diffusion de l'information qu'il recueille. Aujourd'hui un peu plus de 500 ha de carrières de craie ont fait l'objet de levés topographiques précis, à des profondeurs variant de 6 à 30 mètres.

Depuis 1974, le Cerchar a entrepris, à la demande du S.D.I.C.S., dans le cadre de son programme de recherches pour le Service des Techniques du Sous-sol et de l'Environnement industriel, l'étude de la stabilité de ces carrières souterraines. Cette recherche a été conduite en collaboration avec le S.D.I.C.S., avec deux objectifs :

- définir des critères simples de stabilité ou d'instabilité des carrières souterraines;

- définir des moyens de stabilisation des terrains aussi peu "onéreux que possible lorsque cela est nécessaire.

Au cours des quatre premières années, elle a porté principalement sur des carrières exploitées par chambres et piliers où le problème de la stabilité des piliers apparaît comme crucial, et elle a permis de mettre au point des outils, et une méthode de travail qui a été depuis appliquée avec succès, par la même équipe du Cerchar, sur d'autres sites. L'article qui suit présente cette méthode d'étude et les résultats qu'elle a permis d'obtenir sur un exemple.

Cette recherche se poursuit actuellement par la mise au point de nouveaux outils et l'étude des critères de stabilité des carrières souterraines où le risque de ruine apparaît immédiatement comme beaucoup moins lié à la résistance des piliers qu'à l'instabilité potentielle du toit (fontis, carrières exploitées en «bouteilles", etc.). (1)

L'étude de la stabilité d'un ouvrage souterrain se traduit communément par l'appréciation du coefficient de sécurité statique, défini comme le quotient de la charge admissible par le matériau, par la charge régnant dans le massif. La mise en œuvre de cette conception pose un certain nombre de problèmes que nous aborderons dans le cas simple des exploitations par chambres et petits piliers abandonnés, situées à faible profondeur, c'est-à-dire dont le recouvrement est inférieur à 30 mètres [1].

Après une première partie générale, où nous définissons le coefficient de sécurité comme une variable aléatoire, nous présenterons dans une deuxième partie l'étude d'un cas réel où sont confrontées méthode expérimentale et approche théorique.

(1) Texte de M. Dejean, Ingénieur au C.E.R.C.H.A.R. 


\section{Coefficient de sécurité et risque de ruine}

\section{Dispersion des caractéristiques mécaniques des roches}

D'un point de vue pétrophysique (et donc mécanique), les roches se distinguent des matériaux manufacturés obéissant à des normes, dans la mesure oủ elles sont le fruit d'actions multiples, parfois contradictoires, survenues pendant des dizaines, voire des centaines de milliers d'années.

La variabilité tant dans l'espace que dans le temps, est un phénomène qui doit être pris en compte et intégré au calcul d'un projet minier.

A une caractéristique mécanique, résistance à la compression par exemple, correspond une certaine distribution statistique caractérisée par une moyenne $\overline{\mathrm{R}}$ et un écart type $\sigma$. Pour déterminer la résistance limite $R_{L}$ qui sera introduite dans les calculs, il est souvent fait appel à une relation :

$$
R_{L}=\bar{R}-K \sigma
$$

où est donné à $\mathrm{K}$ le sens d'un certain «facteur" de sécurité dont la valeur est fixée "a priori w et résulte, suivant les auteurs, soit de l'expérience minière [2], soit de la pratique statistique [3].

Le calcul du coefficient de sécurité F proprement dit résulte ensuite du quotient de la résistance limite par la valeur des sollicitations exercées. On voit ainsi apparaitre entre le facteur $K$ et le coefficient $F$, une ambiguité que nous essayerons de lever dans le paragraphe suivant.

Les sollicitations prises en compte peuvent être calculées analytiquement et supposées constantes en tous les points de l'ouvrage minier. Elles peuvent aussi être calculées numériquement, par éléments finis par exemple, en tous les points du milieu discrétisé.

Le premier calcul aboutit à un coefficient de sécurité moyen, alors que le second permet de détecter les points singuliers où peut commencer la rupture.

\section{Risque de ruine}

Soit S le niveau des sollicitations. Nous le supposerons tout d'abord constant en tous les points du massif. Par contre, la résistance est supposée dispersée, avec une certaine distribution statistique. Le risque de ruine est la probabilité $\alpha=\operatorname{Prob}\{\mathrm{R}<\mathrm{S}\}$. L'importance de la dispersion a une influence considérable sur le risque [4]. Ainsi, pour les deux distributions représentées sur la figure 1, il correspond à une même sollicitation $\mathrm{S}$ les risques de $\alpha_{1}$ et $\alpha_{2}$ : le risque $\alpha_{2}$ est supérieur au risque $\alpha_{1}$ alors que la résistance moyenne $\bar{R}_{2}$ est plus grande que $\bar{R}_{1}$.

En pratique, ies sollicitations $S$ régnant dans le massif sont variables parce que les dimensions des chambres et des piliers sont elles-mêmes variables. Supposonsles réparties de façon aléatoire suivant une certaine distribution. La figure 10 qui sera analysée dans la deuxième partie fournit un exemple de distribution observée.

Le risque de rupture se déduit alors de la comparaison des deux variables aléatoires $R$ et $S$ : $\alpha=$ Prob $\{S-R>0\}$. Pour un coefficient de sécurité



Fig. 1 Influence de la dispersion sur la détermination du "risque», d'après Coates (4)

donné, $F=1$ par exemple, la probabilité de rupture est la "surface de probabilité" limitée par la droite $\mathrm{R}-\mathrm{S}=0$, sur la figure 2 .

Le coefficient de sécurité, quant à lui, devient le quotient de deux variables aléatoires. Sa distribution de probabilité peut être estimée en calculant les quotients des $n_{1}$ valeurs de $R$ observées par les $n$ valeurs de $S$ déterminées expérimentalement ou bien calculées pour chaque pilier en fonction de ses dimensions et de celles des chambres. La figure 3 représente un exemple de fonction de répartition obtenue ainsi. Elle est, comme nous le montrerons dans la deuxième partie, passible d'un bon raccordement à une loi de probabilité log. normale.

\section{Choix de la sollicitation tolérable}

Pour un matériau et un ouvrage donnés, il importe de préciser laquelle des grandeurs mécaniques suivantes sera prise en compte dans le calcul :

- Rf limite de fluage (résistance ultime),

- Re limite élastique,

- Rc résistance instantanée.

Suivant le type d'exploitation et la nature du matériau, les conceptions varient avec les auteurs. Nous limiterons les considérations qui vont suivre au cas de la craie. En ce qui concerne ce matériau, Rf est toujours largement inférieur à Rc. $0,5 \mathrm{Rc}<\mathrm{Rf}<0,8 \mathrm{Rc}$. Autrement dit, les grandeurs Re ou Rc conduiraient à des calculs optimistes. Cependant, la prise en compte de Rf peut être critiquée d'un double point de vue. D'une part, la détermination expérimentale de Rf pose un certain nombre de problèmes parce que les essais de fluage ne sont pas normalisés [5] et parce qu'ils ne sont pas forcément représentatifs: leur durée est toujours très courte (de quelques heures à quelques mois) et la taille des échantillons n'excède pas en général la dizaine de centimètres, d'où l'intervention probable d'un effet d'échelle. Plus pragmatiquement, d'autre part, certaines exploitations de craie à fort taux de défruitement, où règnent des contraintes largement supérieures à la limite de fluage sont stables depuis plusieurs siècles. Les mesures de déformation effectuées au $1 / 100$ de $\mathrm{mm}$ à Hordain, pendant près de 


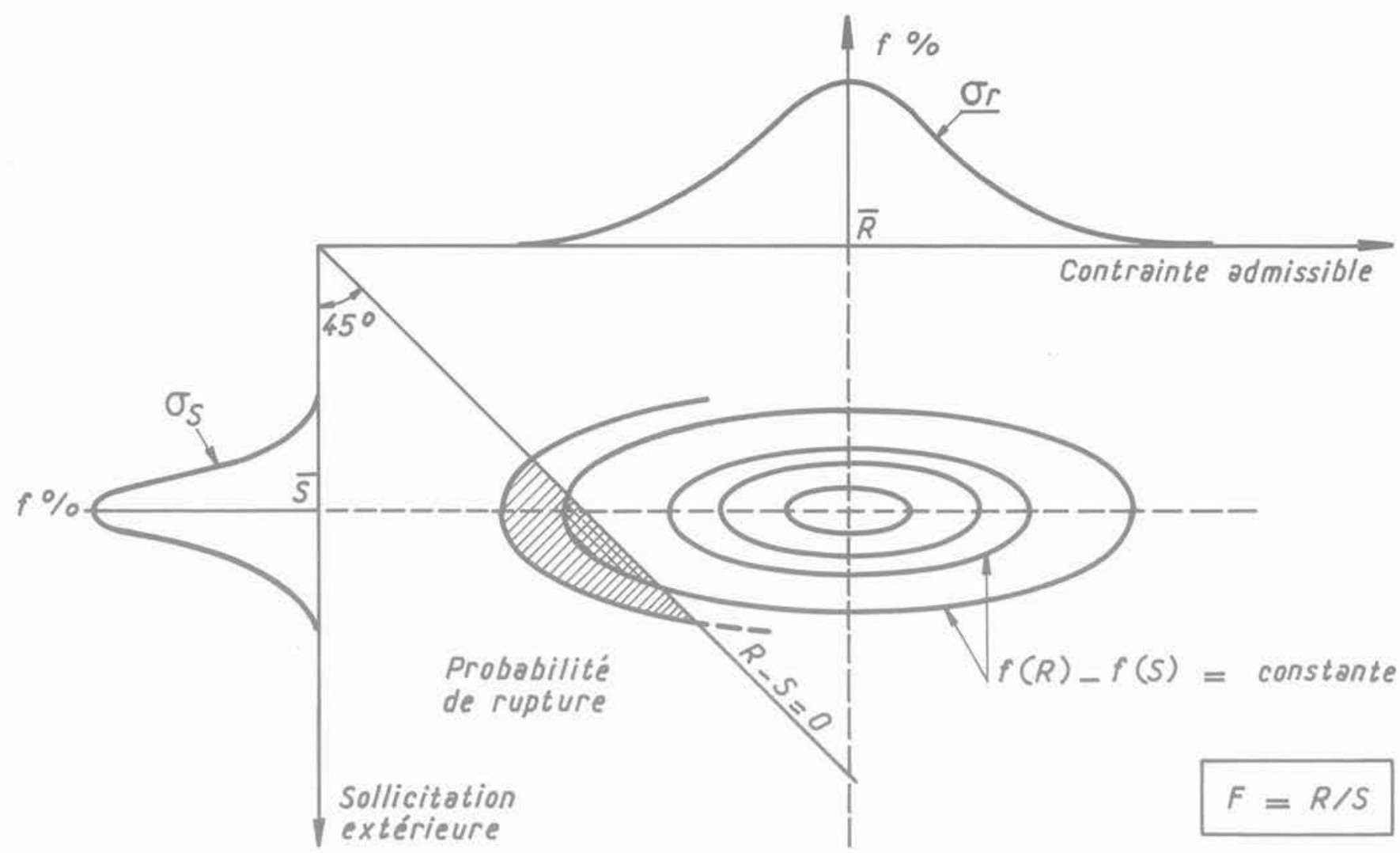

Fig. 2 Surface de rupture pour $F=1$ calculée à partir des distributions des deux variables aléatoires $R$ et $S$

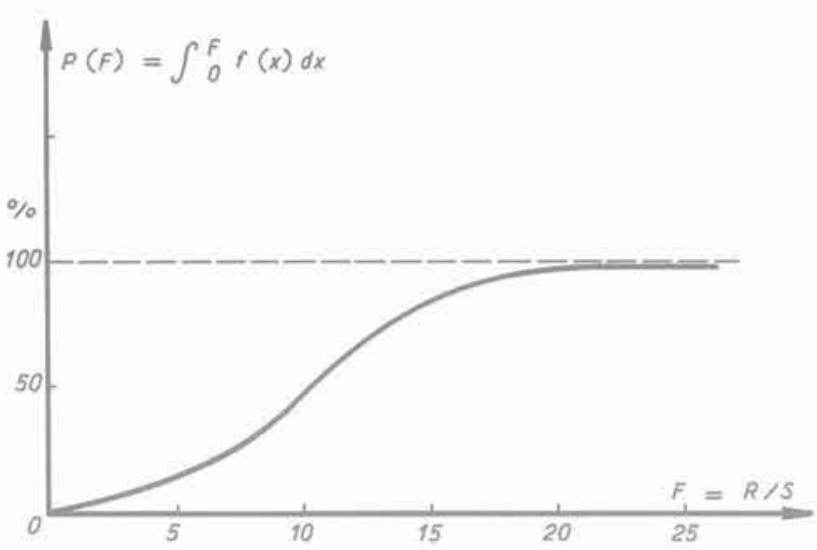

Fig. 3 Fonction de répartition de $F=R / S$

3 années [6] traduisent l'absence de déformations différées.

Les mesures de contraintes en superficie ou en fond de niche, corroborent ces présomptions. Le matériau peut être dégradé en surface et caractérisé par un comportement complexe du type viscoélastoplastique alors que dans le cceur du pilier, la craie garde un comportement "sain" quasiment élastique.

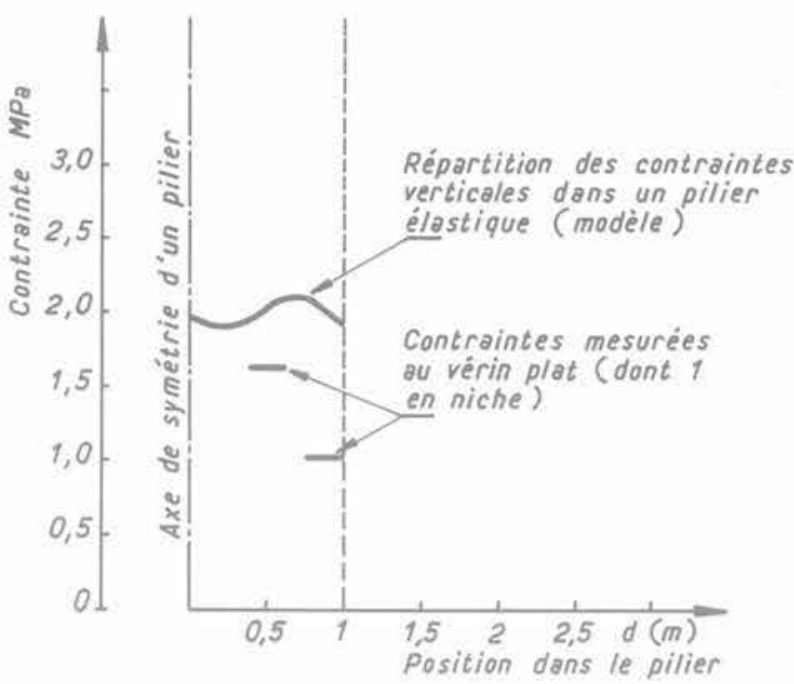

Fig. 4 Comparaison des contraintes verticales calculées par le modèle dans un pilier sain et des contraintes types mesurées dans un pilier fissuré

La figure 4 illustre l'accroissement de la charge mesurée expérimentalement en allant vers le centre du pilier. Ce phénomène du report des contraintes vers l'intérieur du pilier a déjà été observé [7], lors de mesures de contraintes au vérin plat, dans une carrière de craie de la région parisienne. Les piliers carrés de 8 à $10 \mathrm{~m}$ de section ont subi, sous la charge, une accomodation irréversible. 
Ces mécanismes sont identiques, à petite échelle, à ceux mis en évidence dans les tunnels et grandes cavités [8]. On observe rarement des ruptures différées sur les parois d'une excavation dans la craie et il se produit vraisemblablement une adaptation plastique des contraintes autour de la galerie, de sorte que les accommodations tangentielles à la paroi s'adaptent à la résistance ultime de la craie.

Les travaux d'E.D.F. témoignent de la très grande faculté d'adaptation de la craie aux sollicitations, à condition que l'eau puisse être évacuée librement. A Venteuil, les déformations ont été plus faibles que celles calculées par un modèle d'éléments finis dans I'hypothèse élastique $[9,10]$.

De même, à Paluel, la craie de qualité médiocre (3MPa $<\mathrm{Rc}<5 \mathrm{MPa}$ ) a présenté un excellent comportement malgré des contraintes tangentielles égales à $3,4 \mathrm{MPa}$ (mesurées au vérin plat) [11].

Pour la craie saturée, on peut donc choisir un taux de travail supérieur; mais il donnera lieu à des déformations différées, liées [8] aux mouvements de l'eau interstitielle et au comportement visqueux du squelette solide. Or la dissipation des pressions interstitielles est un phénomène lent dans les milieux poreux comme la craie, caractérisés par une faible perméabilité. Si la sollicitation est trop forte et proche de Rc, le temps nécessaire pour obtenir la rupture est court (nul pour $\mathrm{Rc}$ ) et les pressions interstitielles ne sont pas dissipées.

Les propriétés d'accomodation de la craie saturée nous incitent à choisir la limite élastique Re correspondant aux déformations longitudinales, qui se situe généralement entre 0,8 et $0,9 R c$ pour ce matériau.

\section{Les modèles de comportement}

La connaissance de la répartition des sollicitations nécessite un grand nombre de mesures pour être crédible et autoriser une prévision objective.

En ce qui concerne la mécanique des exploitations par petits piliers abandonnés, et surtout à faible profondeur, il semble que l'aspect déterminant soit celui de la charge appliquée sur les piliers et de la répartition des contraintes qui en découle. La réaction du toit et du mur à ces efforts constitue un deuxième aspect dont l'importance est conditionnée par les discontinuités tectoniques (failles, diaclases) et stratigraphiques (diasthèmes, épaisseur du premier banc du toit, etc.).

Les modèles de comportement résultent de la confrontation des données expérimentales (mesure des contraintes essentiellement) et des calculs théoriques. Affinés de proche en proche, ces modèles permettent l'obtention d'une distribution théorique des contraintes, autorisant le calcul d'un coefficient de sécurité $F$.

Expérimentalement, les contraintes sont mesurées par la méthode du vérin plat $[12,13]$ et par celle de la rosette des déformations.

D'un point de vue théorique, la quantification des contraintes comporte 2 volets :

- une analyse globale des efforts qui peut être résolue analytiquement ou numériquement (éléments finis à 2 ou 3 dimensions),

- une analyse détaillée des efforts, qui vise à étudier l'influence de la géométrie des piliers. Pour ce faire, on utilise également la technique des éléments finis.
Tout l'intérêt de ces calculs réside dans leur confrontation «a posteriori " avec l'expérimentation. En effet, la justesse des valeurs numériques utilisées et la validité des hypothèses de départ ne sont jamais assurées.

\subsection{Le type monolithique}

Il est le siège d'accumulation d'énergie élastique, et de reports latéraux des charges exercées.

Le premier banc du toit est très compétent, de grande épaisseur $(0,50$ à 1 mètre) et continu. L'étude des distributions de contraintes peut se faire d'une manière analytique. Ainsi, les calculs menés [14] dans le cas simple d'une dalle carrée encastrée sur les piliers fournissent les valeurs des moments au centre des piliers et au centre de la dalle.

Les résultats ont été largement utilisés [15, 16] tant à moyenne profondeur que pour certaines exploitations situées dans un domaine plus superficiel. Ces calculs permettent des approches similaires en considérant, par exemple, une plaque encastrée ou reposant simplement sur des appuis élastiques (simples ou multiples, etc.).

D'une manière numérique, les modèles tridimensionnels d'éléments finis disponibles permettent la modélisation de plaques de grandes dimensions. Les piliers sont alors représentés par des forces ponctuelles disposées conformément au schéma d'exploitation.

\subsection{Le modèle de l'aire tributaire}

On suppose que la charge des terrains surincombants se répartit intégralement sur les piliers, sans report latéral sur les bordures d'exploitation. Ainsi à 3 dimensions, et dans le cas d'une exploitation horizontale :

$$
\sigma_{p}=\frac{\sigma_{0}}{1-\tau}
$$

$-\sigma_{p}=$ contrainte verticale,

$\sigma_{0}=$ contrainte initiale avant creusement,

$\tau=$ taux de défruitement.

Des paramètres essentiels comme les dimensions de l'exploitation ou des piliers (et notamment la hauteur) ainsi que leurs positions respectives par rapport aux bordures, ne sont pas prises en compte (4).

Cette théorie ne s'applique que lorsque le nombre de piliers est important et, essentiellement, dans les zones les plus centrales.

Dans la pratique, le toit doit être constitué de bancs discontinus assez épais $(0,30$ à 0,50 mètre), à fracturation verticale marquée mais d'orientation variable.

\subsection{Autres modèles}

Entre ces types extrêmes, différents cas sont possibles suivant la nature du toit et les dimensions de l'exploitation.

- Le modèle de blocs assez courant pour des matériaux gélifs et facilement altérables, nécessite des configurations géométriques particulièrement régulières. Ce cas est fréquent dans les exploitations de pierre à chaux, où le matériau très fracturé est emballé dans une pâte calcaire résultant de I'altération.

- Le modèle de poutres, explicité plus en détail dans la deuxième partie, où la structure joue un rôle primordial. 


\section{Conclusion de la première partie}

Le coefficient de sécurité statique défini comme une variable aléatoire est associé à la notion de risque. La connaissance de sa loi de distribution peut être approchée théoriquement ou expérimentalement. Le cas réel qui va être présenté maintenant, a pour but de comparer ces deux approches et d'élaborer une méthode d'investigation utilisable pour des exploitations de surface importante, où le coût de l'approche purement expérimentale serait prohibitif.

\section{Le cas des carrières de Lezennes}

L'extension vers le Sud de l'agglomération lilloise est freinée par une ceinture quasi-continue d'anciennes carrières souterraines parmi lesquelles celles de Lezennes posent de réels problèmes de constructibilité, soit pour l'implantation de bâtiments, soit pour l'extension du réseau routier, d'autant que ce dernier doit répondre à des normes de plus en plus exigeantes. Paradoxalement, ces carrières dont l'exploitation intensive a favorisé l'essor économique d'une grande cité pendant plusieurs siècles constituent aujourd'hui un obstacle à son développement.

Le site proprement dit couvre environ 150 hectares. II a connu plus de 10 siècles d'exploitation par la méthode des catiches et chambres et piliers (figure 5).

\section{Le contexte géologique}

La craie exploitée appartient au Sénonien; la limite Turono-Sénonienne, fixée au premier tun (*) par L. Cayeux, apparaît parfois au mur de la carrière à la faveur d'anciens puits non remblayés. Ces emplacements sont d'ailleurs généralement recouverts par la nappe phréatique qui baigne le pied des piliers.

Au point de vue structural, le site est caractérisé par deux grandes familles de diaclases sub-verticales et sensiblement orthogonales, parfaitement corrélées avec les accidents cassants du dôme du Mélantois.

\section{Caractérisation du matériau}

La caractérisation physique et mécanique du milieu a été effectuée à partir des données relatives à 4 sondages carottés, complétées par des prélèvements superficiels opérés au sein des piliers et du toit.

\subsection{Lithologie}

La succession lithologique observée est représentée sur la figure 6; elle permet de distinguer 5 grands types de faciès, dont la craie piquetée qui a été exploitée. Cette roche appelée également craie grise de Lezennes présente des grains de glauconie, visibles à l'œil nu, dispersés dans la matrice crayeuse de manière aléatoire.

(") Le premier tun est un niveau phosphaté et induré d'une cinquantaine de centimètres d'épaisseur.

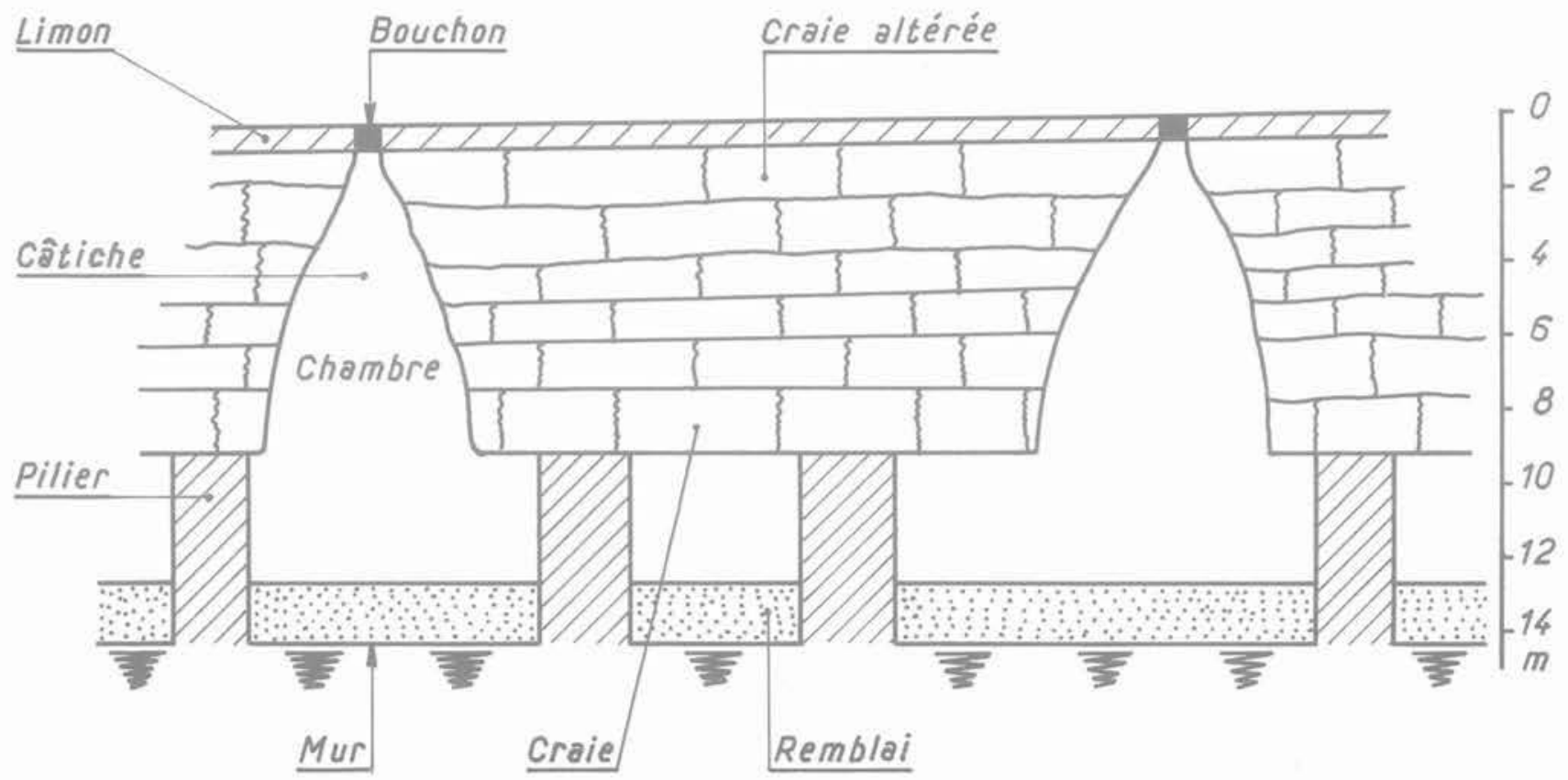

Fig. 5 Exploitation en «catiche» de la région de Lezennes 

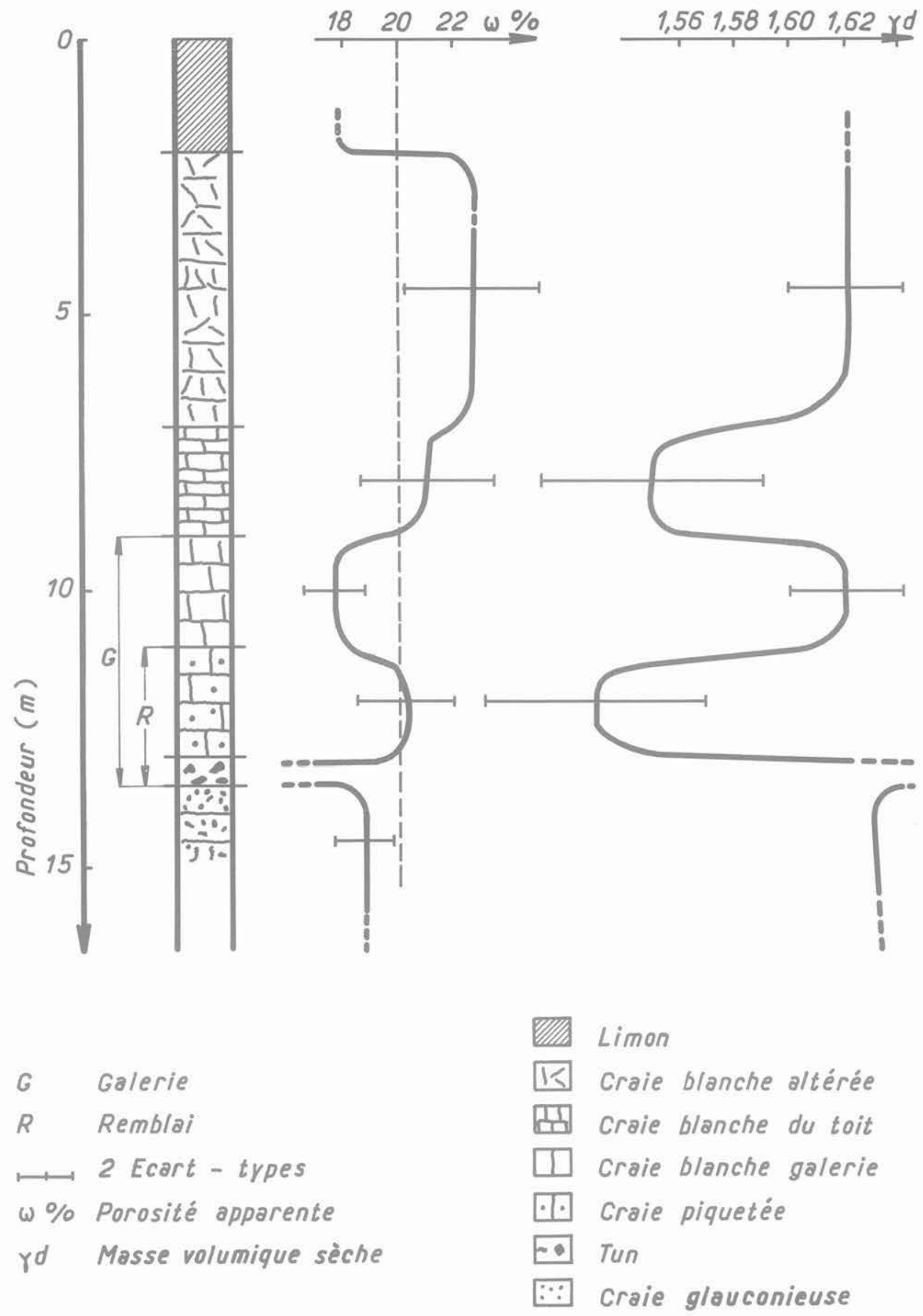

Fig. 6 Succession lithologique et variation des paramètres physiques au sein de différents horizons (porosité apparente et masse volumique sèche) 


\subsection{Propriétés physiques}

Les propriétés physiques traduisent une relative homogénéité du matériau, à l'exception du premier tun (*). D'une manière générale, l'altération et la fissuration superficielles ont pour effet d'accroître la porosité apparente et le poids spécifique (craies blanches du Coniacien supérieur). La figure 6 représente les variations de ces grandeurs avec la

(*) Les valeurs fournies pour ce niveau sont d'ailleurs purement indicatrices, à cause de son hétérogénéité. profondeur; elle fait apparaître une coupure assez nette au sein du niveau exploité entre la craie blanche (pierre à chaux) et la craie piquetée (craie à bâtir).

\subsection{Caractérisation géomécanique}

D'une manière générale, les craies de Lezennes sont isotropes et saturées, ou proches de la saturation. Le matériau constituant les piliers présente une grande dispersion et des distributions de type bi-modal (fig. $7 \mathrm{a}$ et 7 b). Par ailleurs, l'étude de l'effet d'échelle a montré que la résistance instantanée était indépendante de la taille des éprouvettes.

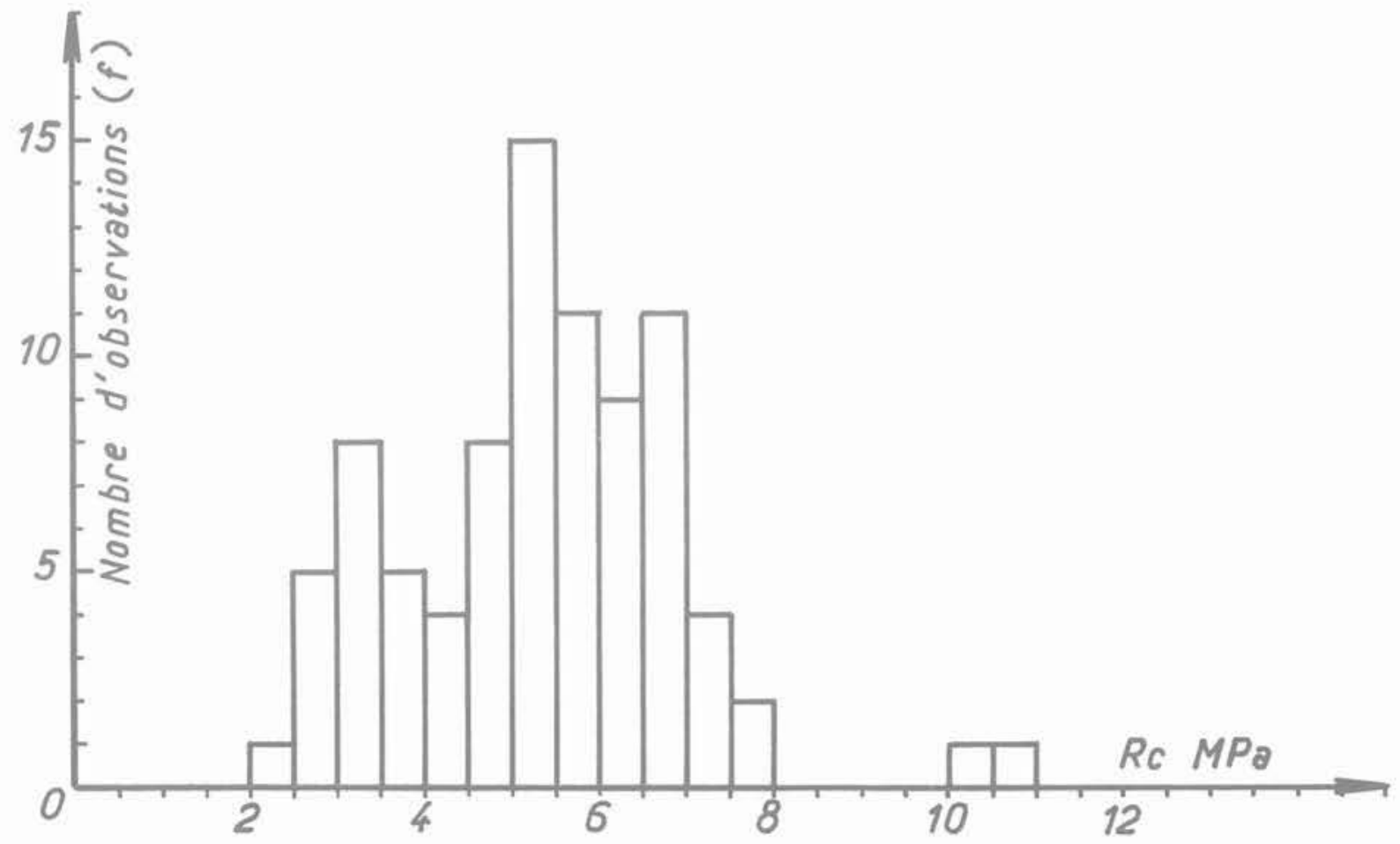

7a Histogramme de la résistance à la compression

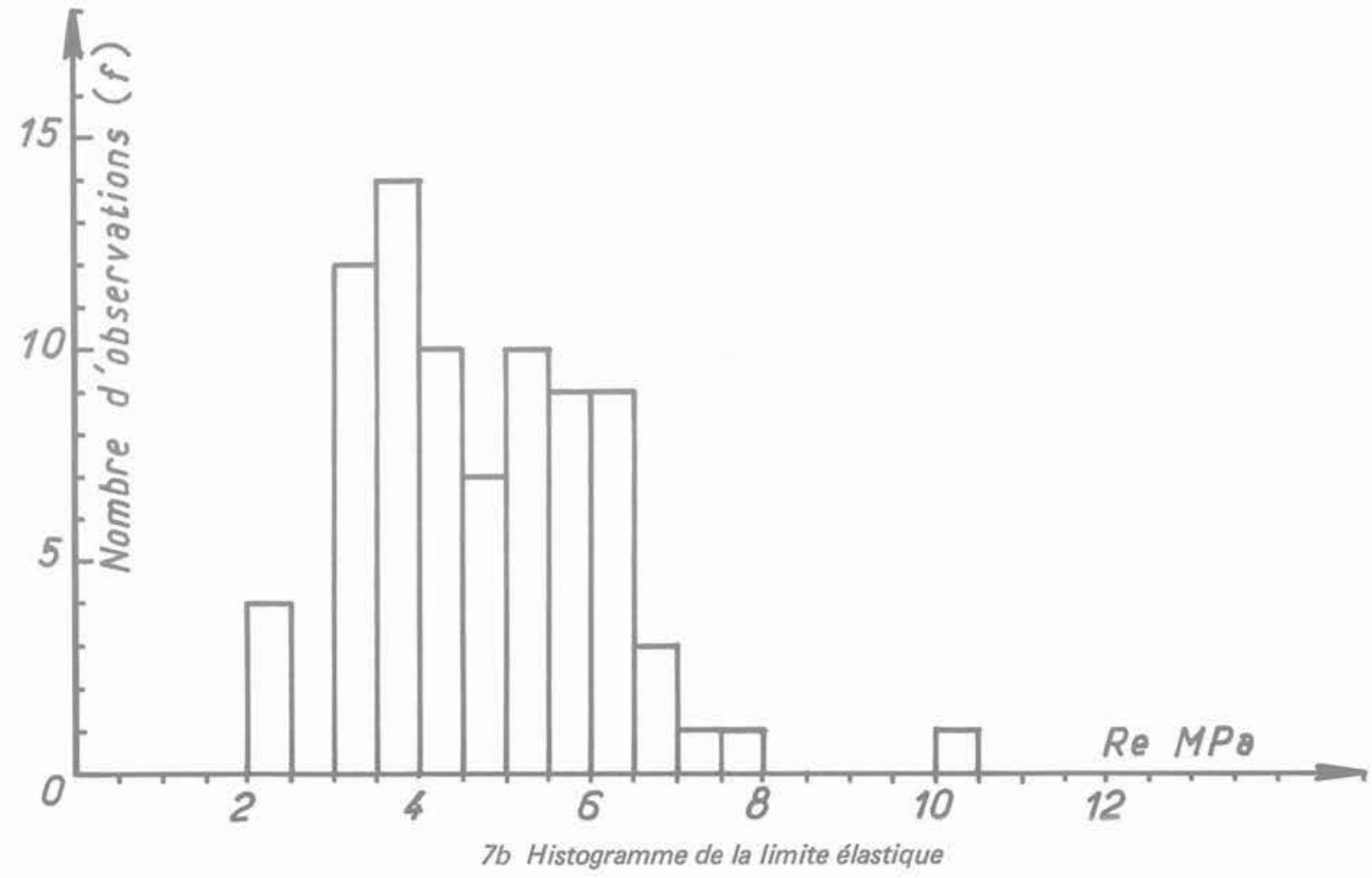

Fig. 7 Lezennes - Craie saturée - $\emptyset 76 \mathrm{~mm}-$ Elancement 2 


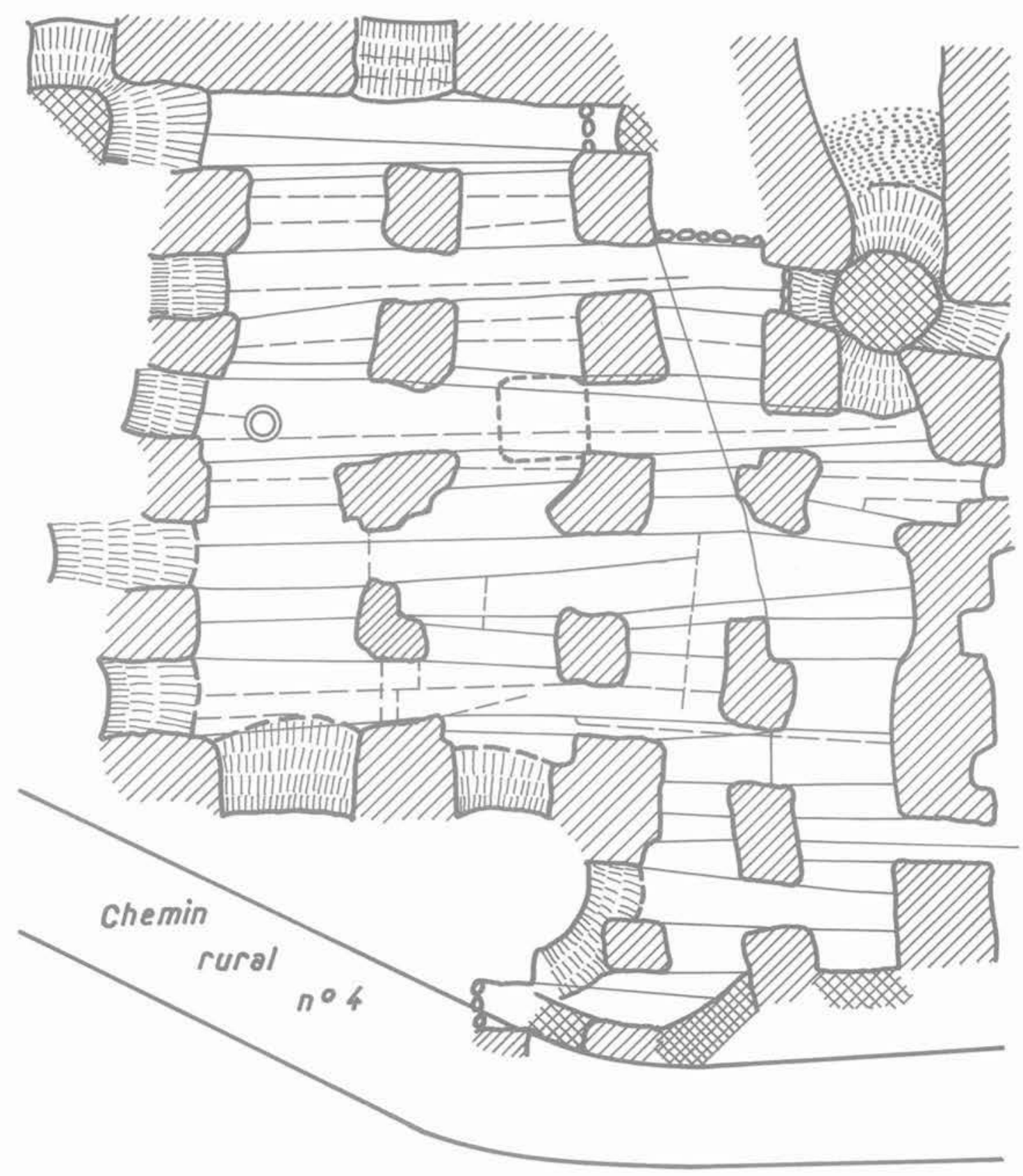

Ech.

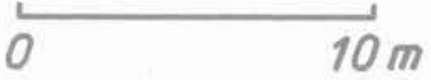

Fig. 8 Carrières de Lezennes (C.D. 146). Puits d'accès $n^{\circ} 16$. Principales fractures naturelles

Les mesures du module de déformation traduisent l'isotropie du milieu. On vérifie que les modules sont statistiquement égaux quels que soient l'emplacement et l'orientation de l'essai. La valeur moyenne, retenue pour l'ensemble des calculs est $4200 \mathrm{MPa}(\mathrm{s}=1350)$.

En conclusion le matériau de Lezennes a toutes les caractéristiques d'une craie de qualité moyenne, tant du point de vue physique que du point de vue mécanique.
Cependant, compte tenu des sollicitations mécaniques moyennes prévisibles, il semble que le matériau soit largement en deçà des charges critiques, du moins au niveau des piliers. En effet, le critère le plus pessimiste conduit à un rapport de 4,75 entre les contraintes tolérables et les contraintes régnant dans le massif ("*).

(“*) Le taux de défruitement moyen qui est égal à $70 \%$ conduit à une contrainte de l'ordre de 0,6 MPa. 


\section{Structure}

A l'échelle générale du site, les bancs sont subhorizontaux. Mais localement des stratifications obliques en biseau ou en courbe contrastent avec la régularité habituelle de la série. L'épaisseur moyenne des niveaux supérieurs est de 30 à $40 \mathrm{~cm}$ alors que les niveaux inférieurs (Banc du roux et Banc du tun) sont plus épais (de 50 à $80 \mathrm{~cm}$ ).

Les discontinuités structurales revêtent une importance particulière du point de vue mécanique. Elles sont caractérisées non seulement par une orientation très constante mais aussi par une grande régularité.

Perpendiculairement à la direction Nord-Sud, on trouve en moyenne une diaclase tous les $90 \mathrm{~cm}$ (écart type de $50 \mathrm{~cm}$ ), la loi de distribution étant log normale. En revanche, perpendiculairement à l'axe Est-Ouest les discontinuités sont abondantes mais d'extension moindre.

Les carriers, très naturellement, ont orienté le schéma d'exploitation suivant la direction principale de la fracturation (fig. 8). Chaque pilier est bordé latéralement par 2 diaclases E.W. quasiment planes et sub-verticales. Les discontinuités au sein du toit ont été mises en évidence par une campagne de relevés endoscopiques. Le premier banc du toit fait en moyenne $30 \mathrm{~cm}$ d'épaisseur et le nombre de discontinuités observées sur les 2 premiers mètres est relativement constant et indépendant de l'emplacement du sondage. D'une manière générale, on rencontre une fracture (ouverte ou fermée) tous les $10 \mathrm{~cm}$.

En conclusion, le milieu rocheux est profondément discontinu :

- les piliers sont constitués de blocs de petites section $(0,90 \times 0,30$ mètre en moyenne),

- le toit est un assemblage de blocs linéaires de $0,10 \times 0,90$ de section, maintenus par frettage et appuyés de proche en proche sur les piliers.

\section{Géométrie de l'exploitation}

Sur une superficie d'environ 4 hectares couvrant l'ensemble des plans au 1/200 actuellement disponibles a été effectuée une analyse statistique des principaux paramètres de l'exploitation :

- hauteur des galeries: $h$,

- taux de défruitement: $\tau$,

- section moyenne des piliers : $s$,

- nombre moyen de côtés par pilier : $n$.

Les valeurs moyennes et les dispersions sont citées dans le tableau de la figure 9 .

\begin{tabular}{l|c|c}
\cline { 2 - 3 } & moyenne & écart type \\
\hline $\begin{array}{l}\text { Hauteur des galeries } \\
\text { Taux de défruitement }\end{array}$ & $2,10 \mathrm{~m}$ & 0,52 \\
$\begin{array}{l}\text { Section moyenne } \\
\text { des piliers }\end{array}$ & 10,56 \\
$\begin{array}{c}\text { Nombre moyen } \\
\text { de côtés par pilier }\end{array}$ & $18,21 \mathrm{~m}^{2}$ & \\
\hline
\end{tabular}

Fig. 9

\section{5 Élaboration d'un modèle de comportement}

Un modèle de comportement doit notamment prendre en compte les paramètres fondamentaux que sont :

- les diaclases et leurs effets sur les reports de charges,

- le taux de défruitement considéré d'un point de vue probabiliste,

- les hétérogénéités locales, naturelles ou artificielles.

Les discontinuités verticales E.W. affectent le toit comme les piliers. Le toit, quant à lui, peut être considéré dans l'hypothèse la plus plausible comme la juxtaposition de poutres minces maintenues par frettage, d'une part, et par les appuis des piliers, d'autre part.

L'approche expérimentale sera basée sur la mesure des contraintes. Leur confrontation aux résultats des calculs théoriques permettra d'affiner et de préciser le modèle.

\subsection{Mesures des contraintes}

L'ensemble des mesures réalisées se décompose en 64 mesures au vérin plat dont 42 mesures de la contrainte verticale et 22 mesures par la méthode de la rosette des déformations.

L'accord entre les deux méthodes a été vérifié par des essais en des points singuliers.

Nous allons rechercher la distribution théorique la mieux corrélée avec la distribution expérimentale des contraintes verticales (fig. 10) qui est log normale.

\subsection{Choix d'un modèle}

Une étude effectuée par la méthode des éléments finis a permis d'analyser l'influence des discontinuités ouvertes ou fermées sur la répartition des contraintes au sein des piliers (fig. 11). Les principaux résultats sont les suivants:

- si la diaclase bordant le pilier est fermée, il se développe des efforts de cisaillement qui se traduisent par une dissymétrie peu importante mais notable au niveau de la section médiane;

- au contraire, si la diaclase est ouverte, aucun report de charge n'est possible et la dissymétrie est plus importante (le facteur de concentration atteint $2,15)$.

Ainsi est-il possible de calculer les distributions théoriques suivant différents types de modèle:

1) le modèle de l'aire tributaire,

2) le modèle de poutres solidaires (diaclases fermées),

3) le modèle de poutres libres (diaclases ouvertes),

4) un modèle intermédiaire, dérivé des deux précédents par combinaison.

L'obtention du dernier type nécessite quelques éclaircissements. On suppose que le champ expérimental est traversé par une proportion $x$ de diaclases fermées et donc $(1-x)$ de diaclases ouvertes. Les contraintes mesurées résultent de cette combinaison. Le meilleur ajustement correspond au type mixte, où I'on considère que $70 \%$ des diaclases sont fermées et $30 \%$ sont ouvertes.

Sur la figure 12, sont portés les histogrammes des contraintes verticales obtenues suivant ces différents 


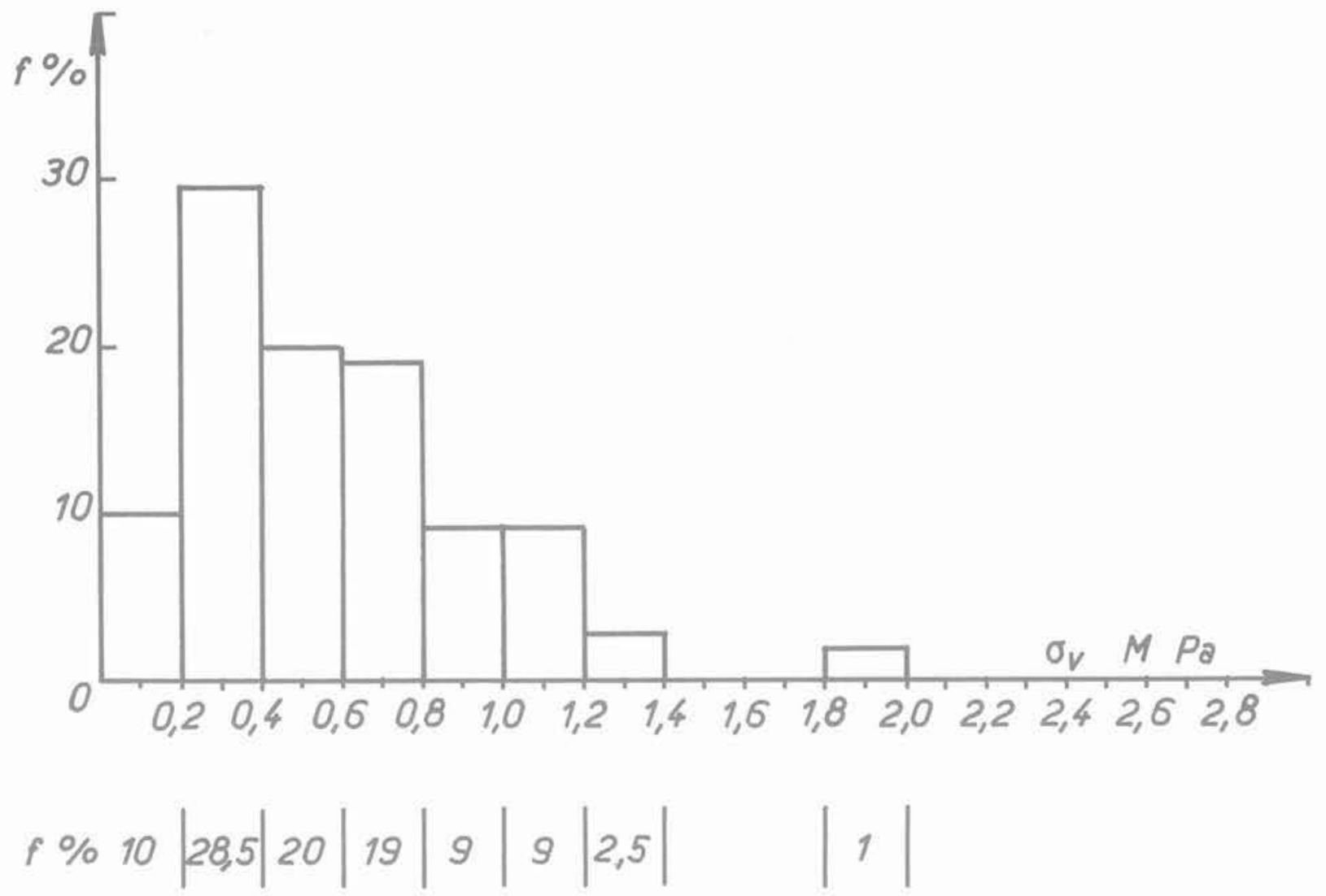

Fig. 10 Histogramme des contraintes verticales mesurées par les deux méthodes

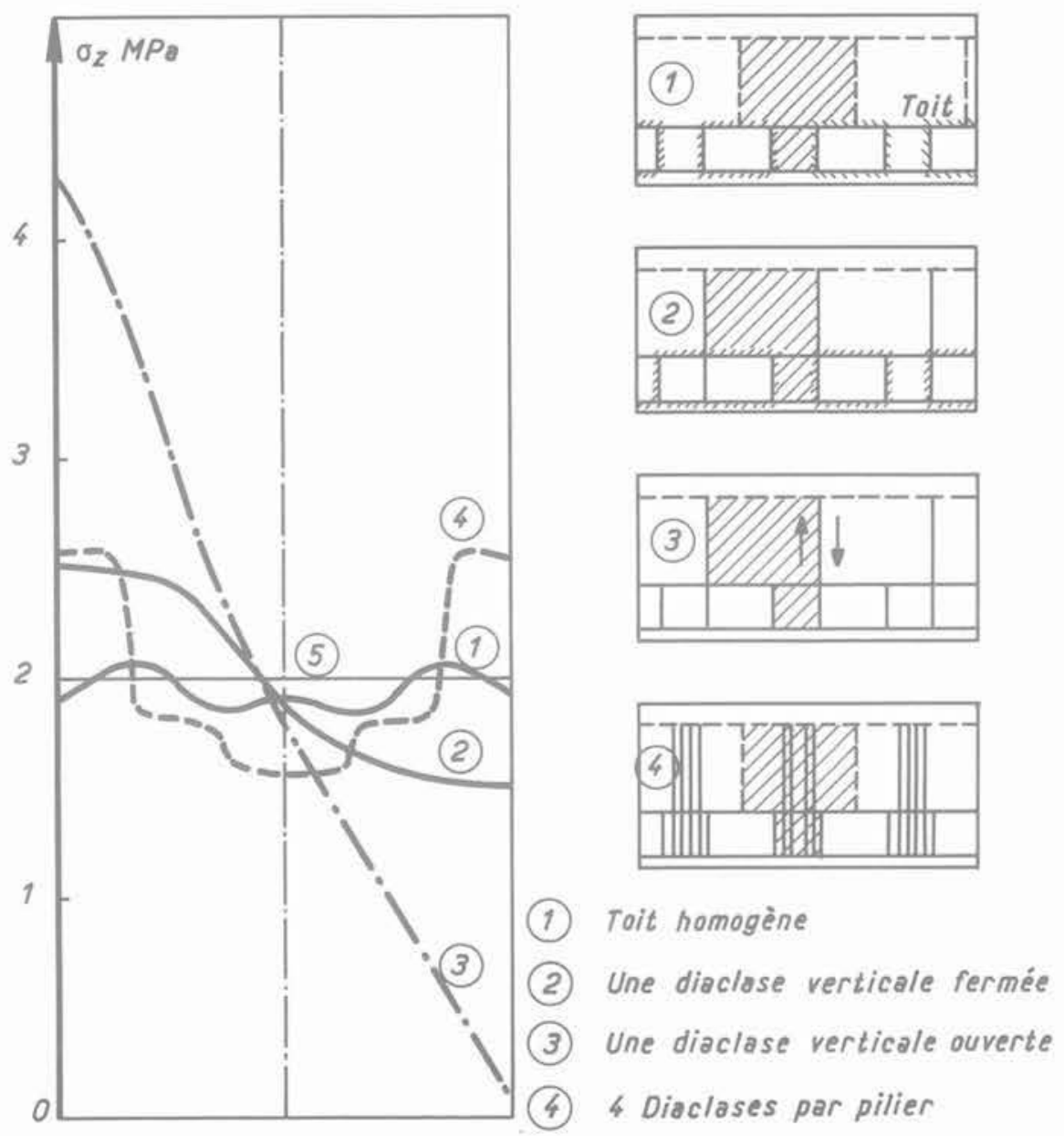

Position \&̀ l'intérieur du pilier

Fig. 11 Distribution de $\sigma_{V}$ au sein d'un pilier suivant différentes hypothèses ( $T=90 \%$ ) 


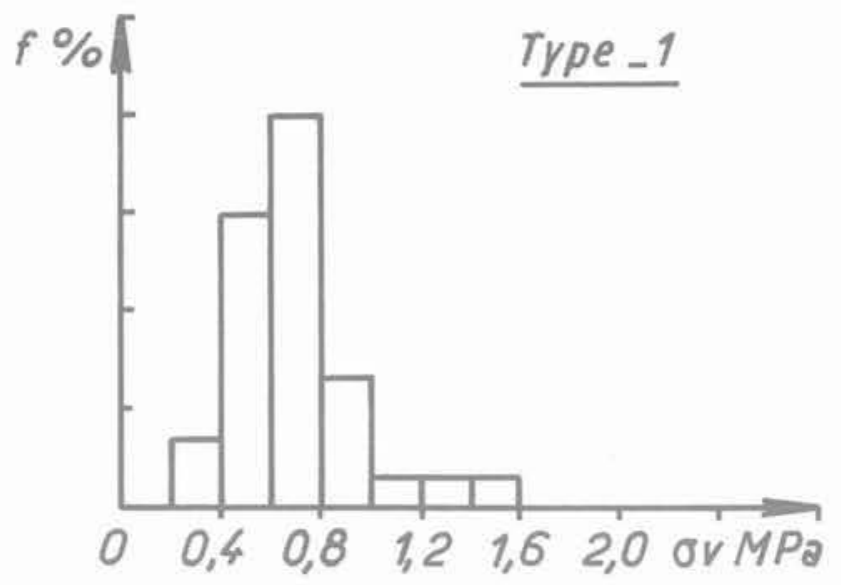

a Distribution theorique

Modèle de l'aire tributaire
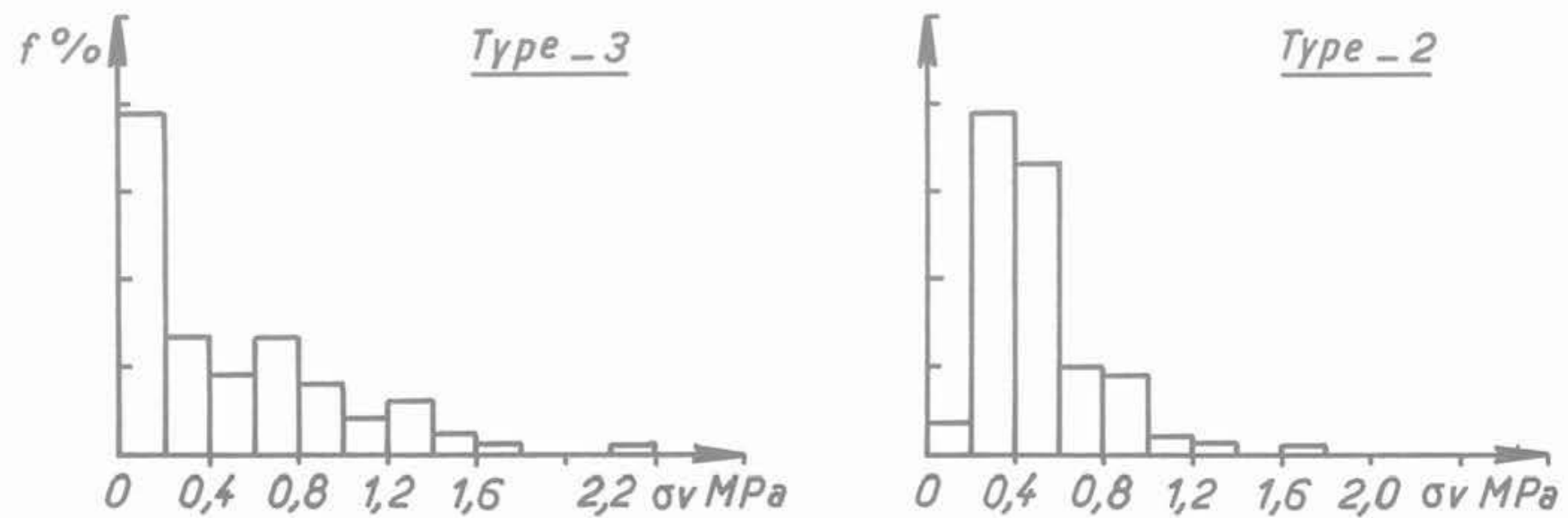

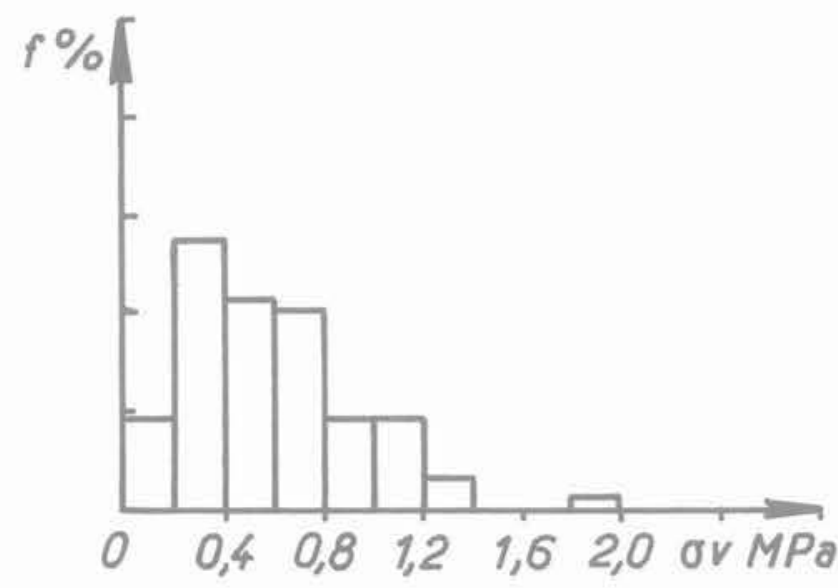

d Distribution expérimentale

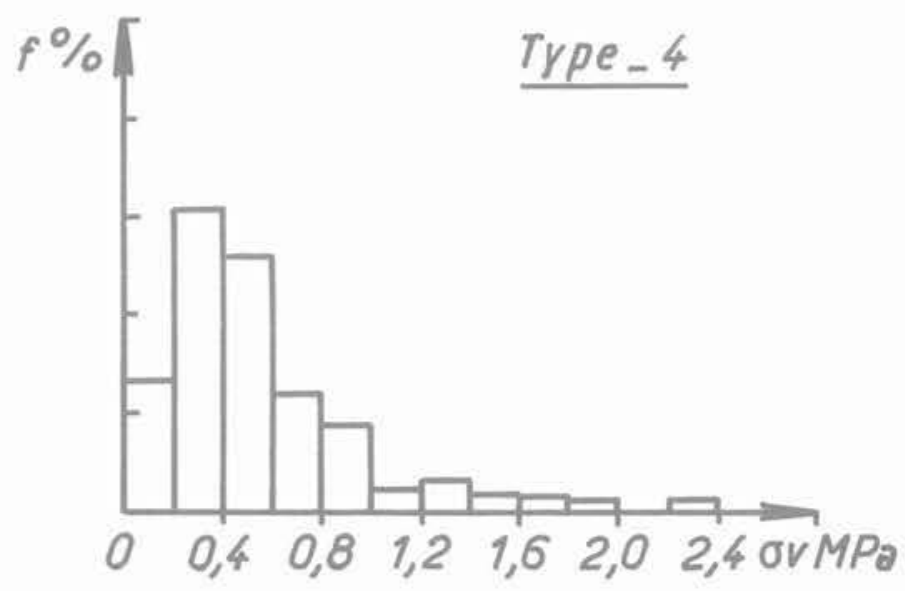

e Distribution théorique suivant le modèle adopté

Fig. 12 Lezennes - Zone témoin - Comparaison de la distribution expérimentale des contraintes verticales avec les distributions obtenues suivant différents modèles (A l'échelle de la zone étudiée) 


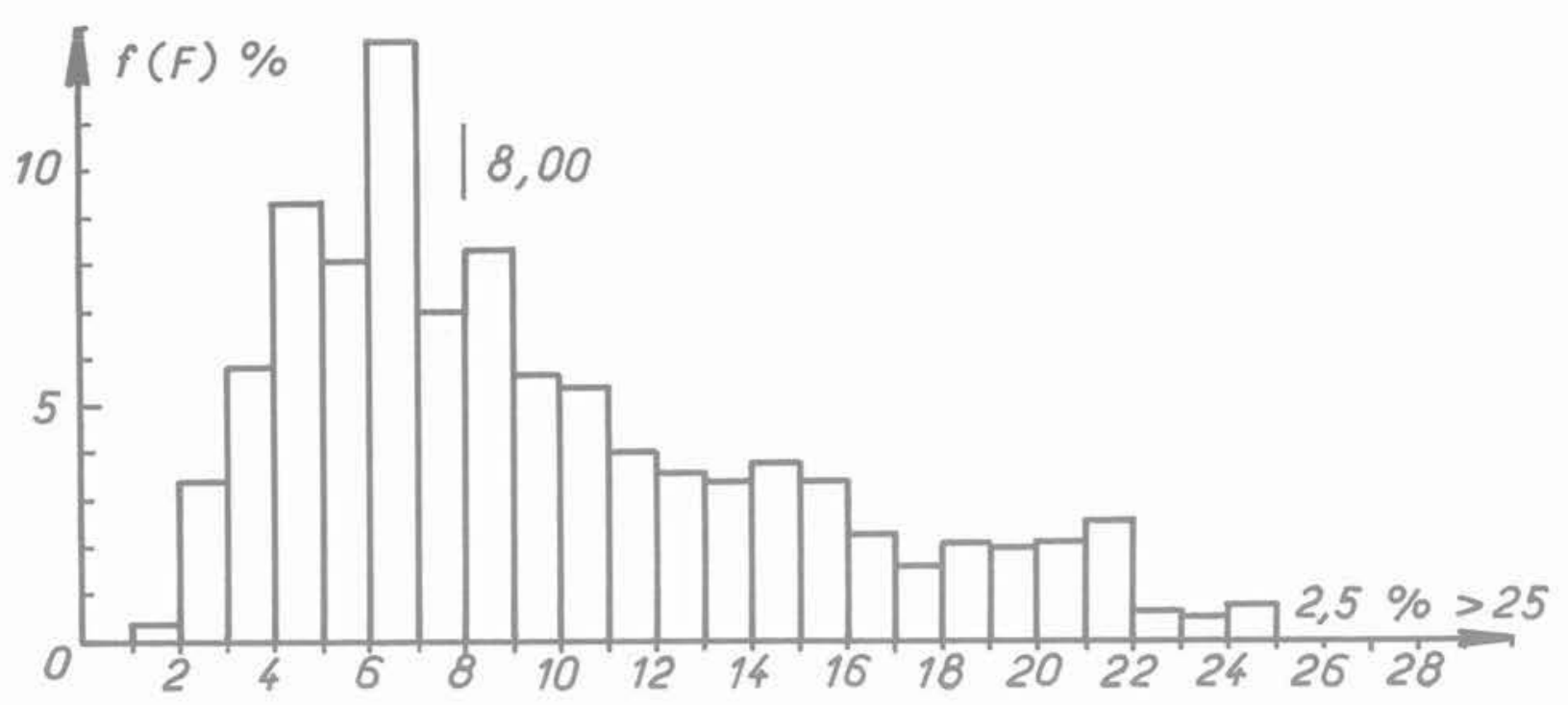

a Distribution expérimentale de $F=R / S$ dans la zone pilote

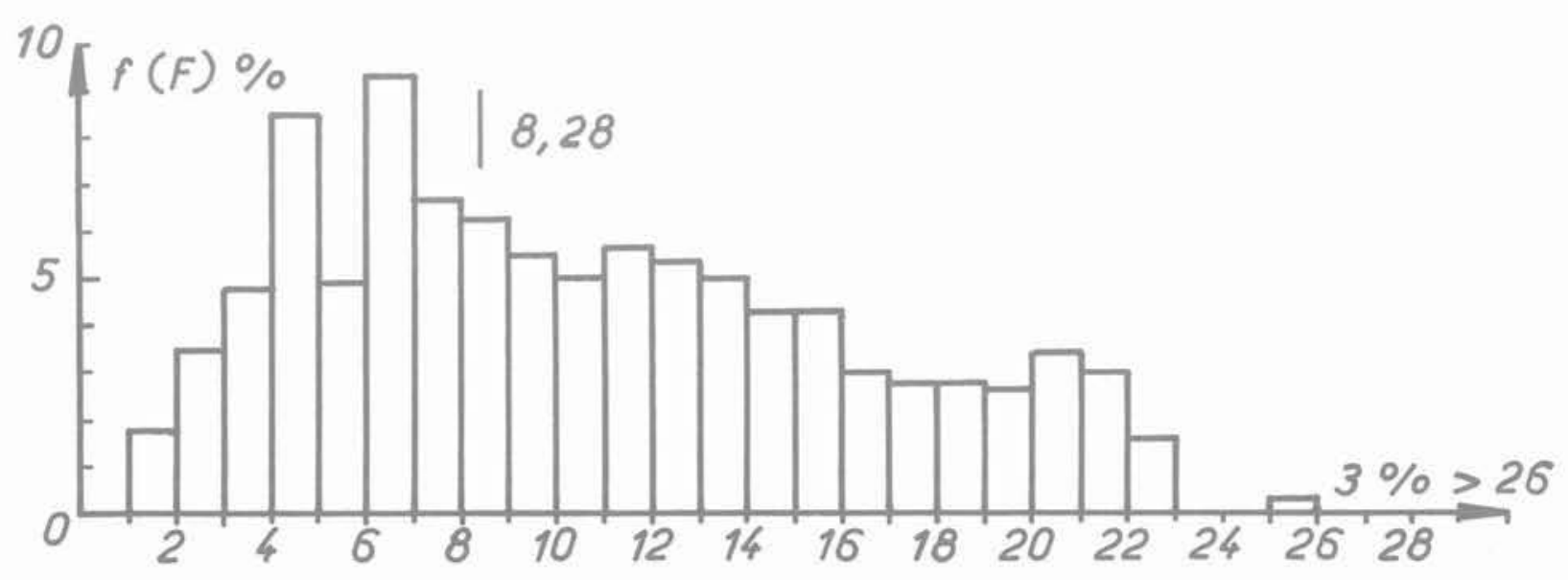

$b$ Distribution theorique de $F=R / S$ dans la zone pilote

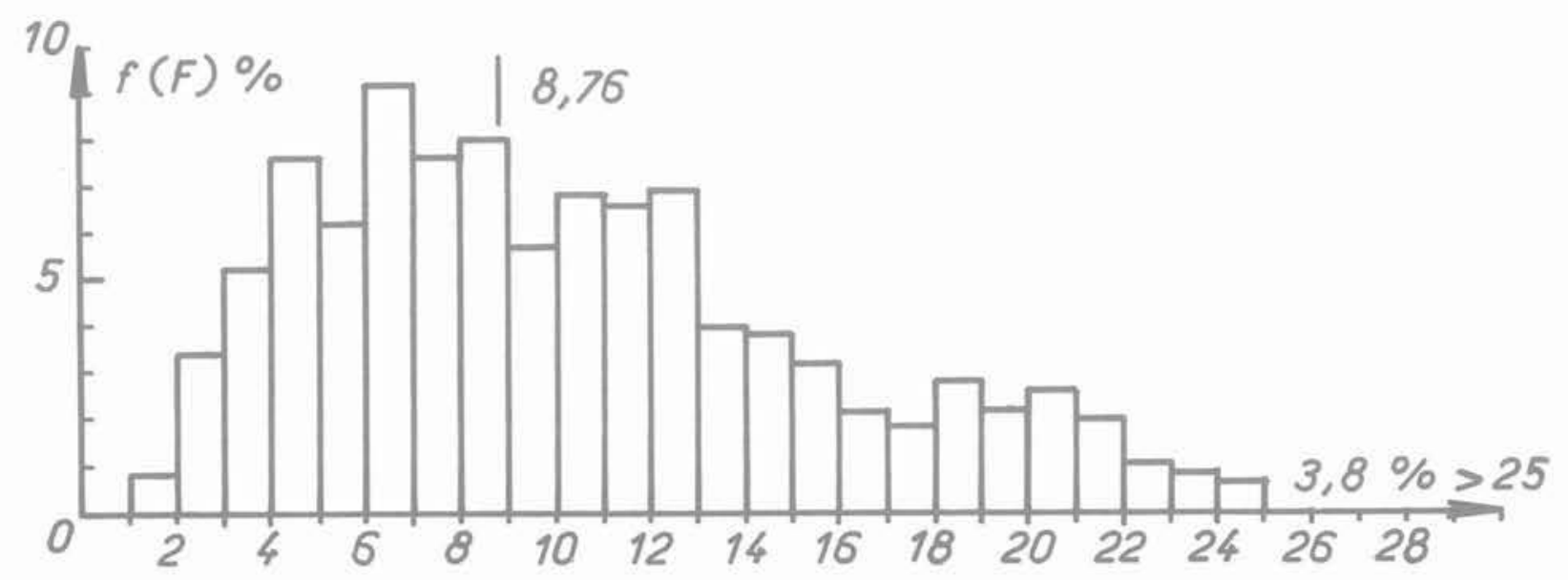

c Distribution théorique de $F=R / S$ pour l'ensemble de la carrière ( $C D$ 146)

(A l'échelle de la carrière)

Fig. 13 Détermination de $F=R / S$ 
modèles, ainsi que la distribution expérimentale. On peut constater que les résultats concernant le modèle de l'aire tributaire sont fort éloignés des résultats expérimentaux.

\subsection{Généralisation à l'échelle de la carrière}

Le modèle de comportement élaboré au sein d'une zone pilote a été testé en trois zones singulières où sa conformité aux mesures expérimentales a été vérifiée.

A l'échelle globale du site, nous avons fait l'hypothèse que $70 \%$ des diaclases étaient fermées et $30 \%$ ouvertes. Ceci n'est qu'un simple modèle et ne signifie nullement qu'une diaclase est ouverte (ou fermée) sur toute sa longueur. Telle ou telle discontinuité au contraire est vraisemblablement ouverte ici et fermée ailleurs. S'il en était autrement, aucun effort latéral ne pourrait véritablement se transmettre.

Moyennant cette hypothèse relative aux diaclases et toujours dans l'optique d'un modèle de poutre, la distribution théorique des contraintes verticales a été calculée à partir des plans aư 1/200 de Lezennes préalablement discrétisés en parcelles élémentaires. La comparaison des contraintes admissibles par le matériau aux sollicitations régnant dans le massif permettra ultérieurement l'élaboration de la distribution théorique du coefficient de sécurité. Toutefois, l'interprétation statistique des distributions à l'échelle globale sous-entend la répartition aléatoire des zones fortement défruitées, ce qui n'est pas vérifié. Aussi faut-il se garder de tirer des conclusions sur la stabilité générale d'un site avant d'avoir considéré les distributions locales dans certaines zones singulières qui méritent une attention particulière.

\subsection{Définition du coefficient de sécurité}

La démarche adoptée dans le cadre de cette étude repose sur l'analyse des contraintes au sein des piliers. Aussi l'évaluation de la probabilité des risques que nous allons tenter, se situe nécessairement à une échelle globale, et exclut les effondrements locaux, du type fontis. La détermination du coefficient de sécurité $F$, ou plutôt de sa distribution car $F$ est considéré comme une variable aléatoire, repose sur le calcul des quotients des $n_{i}$ valeurs de la résistance élastique par les $n_{i}$ valeurs des contraintes, soit mesurées, soit calculées.

Dans un premier temps, seront présentés les 2 types de distribution auxquelles nous sommes arrivés, ce qui débouchera sur la quantification des risques. Nous généraliserons ensuite ces résultats à l'ensemble de la carrière en utilisant la distribution théorique des contraintes proposées figure 12 .

Les deux distributions de $F$ (fig. $13 a$ et $13 b$ ) sont similaires. $F$ est toujours supérieur à 1 , et rarement inférieur à $2(0,4 \%$ et $1,8 \%)$. Les graphiques tracés sur papier gausso-logarithmique (fig. $14 \mathrm{a}$ et b) montrent que ces distributions se raccordent très bien à une loi de probabilité log-normale. II correspond à ce modèle, les valeurs remarquables suivantes:

- Le risque de ruine, c'est-à-dire la probabilité pour que le coefficient de sécurité soit inférieur à 1 . est inférieur à 1/1000;

- la valeur du coefficient de sécurité correspondant à un risque de $1 \%$ est légèrement supérieure à 2 .

Avant de commenter ces résultats, étudions la répartition de $\mathrm{F}$ à une échelle globale.

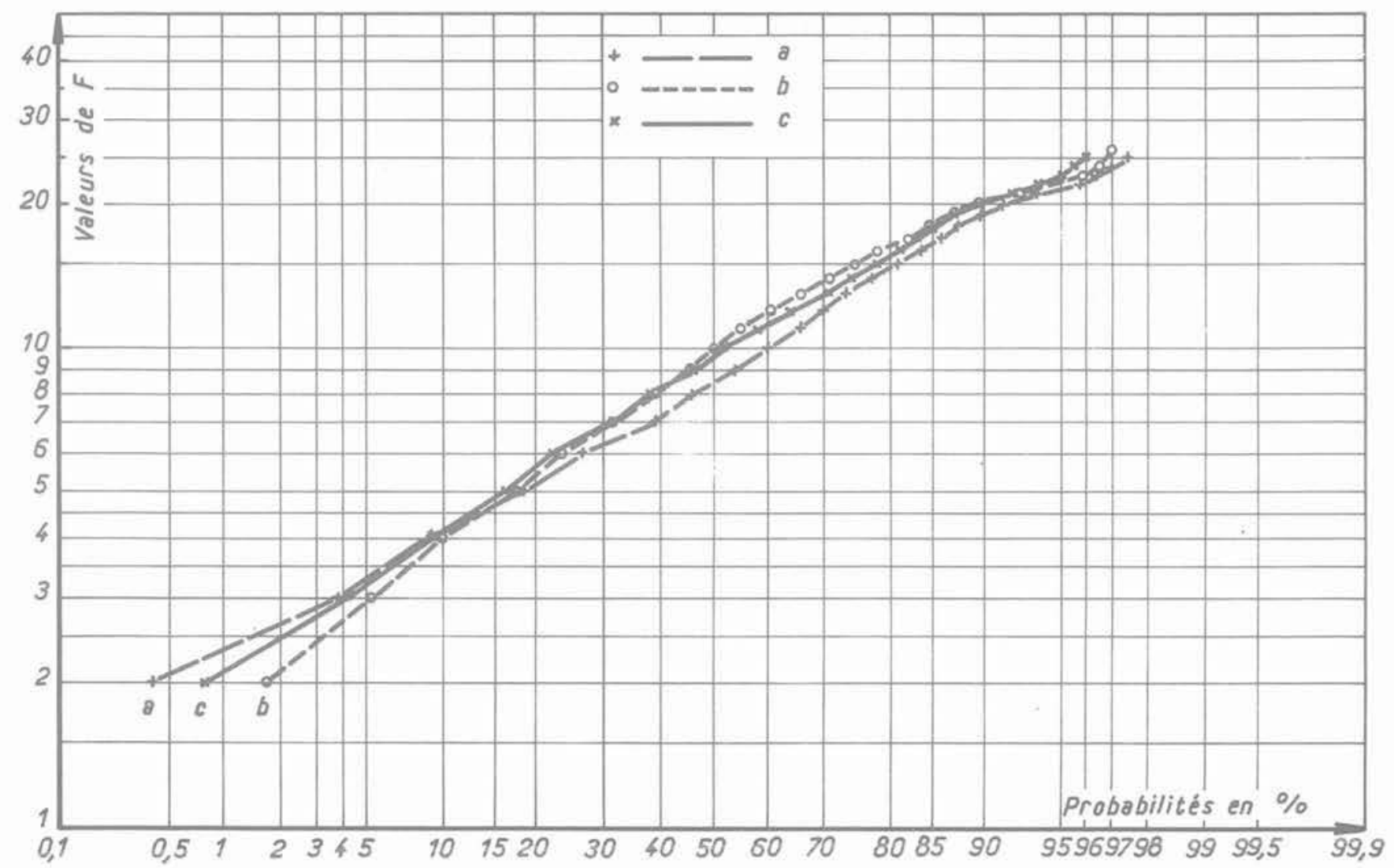

Fig. 14 Raccordement des distributions de $F$ à un modèle log - normal 
Les résultats sont tout à fait comparables. Non seulement l'histogramme est similaire à ceux de la zone pilote mais le modèle précèdent reste tout à fait adapté (fig. $14 \mathrm{c}$ ).

\section{Commentaires et conclusions}

L'évaluation du risque a été abordée successivement sous un angle purement expérimental puis d'une manière théorique. Les résultats obtenus sont convergents. Cependant il arrive qu'ils soient en contradiction avec des observations particulières.

C'est ainsi qu'il existe des zones très sollicitées, caractérisées par un fort taux de défruitement, où certains piliers sont rompus $\alpha$ en diabolon. Ce phénomène traduit l'existence de reports de charges, latéralement, qui peuvent atteindre, suivant le modèle proposé, $25 \%$ de la charge exercée par les terrains surincombants. Cela nous amène à préciser que deux points fondamentaux ont été négligés dans cette étude :

1) Au sein des piliers, la répartition des charges verticales varie avec la section considérée (cf. chapitre 4).

Le contact toit-pilier par exemple est beaucoup plus sollicité que la section moyenne étudiée notamment du point de vue des contraintes de traction.

2) Les reports de charges (25\% au total) transmis latéralement se répercutent en des zones singulières, et probablement dans le haut toit, ou au cœur des piliers, zones non expérimentées. Ces surcharges n'apparaissent donc pas dans la probabilisation.

Le premier point ne constitue pas à notre sens un handicap majeur, du fait des configurations géométriques spécifiques des piliers : les faces Nord-Sud bien qu'anguleuses sont toutes bordées par des diaclases et les concentrations de contraintes sont bien moindres que celles que nous avons proposées dans le cas d'un milieu continu (fig. 11).

Les faces Est-Ouest, quant à elles, présentent toujours un grand rayon de courbure traduisant le souci permanent des carriers de soutenir les longues poutres par un effet d'arche. Or, il est établi, et bien connu, que cette géométrie est particulièrement favorable au niveau de la stabilité.

Enfin, il n'est pas démontré que l'amorce de la rupture en un point singulier entraîne à terme la ruine totale du pilier. Les faits expérimentaux vont même à l'encontre de cette idée un peu sommaire qui néglige, entre autres, la cohésion résiduelle d'un matériau rocheux, même très fracturé. Ainsi, les piliers dits "ruinés" gardent toujours une portance résiduelle que nous estimons voisine de la moitié de la résistance ultime.

En revanche, le problème des reports de charge apparaît * a priori » beaucoup plus épineux et délicat à aborder, tant expérimentalement que théoriquement. En l'état actuel de nos connaissances, le seul élément de réponse réside dans la détermination des zones suspectes, détermination basée sur les critères suivants :

$-\tau>80 \%$,

- longueur d'exploitation $>35$ mètres,

- petits piliers plus ou moins alignés.

La situation des catiches doit être envisagée. Elles contribuent d'une part aux relâchements des contrain- tes latérales et, par là-même, diminuent l'efficacité du frettage. Elles provoquent, d'autre part, des concentrations de contraintes en des piliers singuliers (comme en témoigne une mesure proche de $2 \mathrm{MPa}$. L'ensemble de ces questions ne pourra être véritablement résolu qu'à l'aide de méthodes expérimentales adaptées comme par exemple des mesures de contraintes par surcarottage.

En conclusion, les arguments traduisant la stabilité à l'échelle globale sont suffisamment nombreux pour permettre un diagnostic optimiste. L'effondrement généralisé brutal est «a priori » exclu. En effet, la stabilité des piliers est considérée comme acquise. De plus, l'état de fissuration du toit, tant verticalement qu'horizontalement, s'oppose à l'accumulation d'énergie.

En revanche, les effondrements locaux, les fontis restent et resteront des événements bien difficiles à prendre en compte. Les effondrements de 1976, dus à la rupture d'une canalisation d'eau, nous rappellent qu'en matière de géotechnique il $y$ a toujours l'inattendu.

\section{Références bibliographiques}

[1] J. Bonvallet. Critères de stabilité des exploitations souterraines à faible profondeur. Application au cas des carrières souterraines du Nord.

Juin 1978, Thèse Docteur-Ingénieur Nancy-I. N.P.L.

[2] E. Tincelin. La détermination des caractéristiques mécaniques des massifs rocheux à partir des essais en laboratoire.

Annales des Mines, Février-Mars 1975.

[3] J. P. Josien. - M. Dejean. «Influence de la dispersion des caractéristiques mécaniques des terrains sur la stabilité à long terme des excavations souterraines par petits piliers. "

Cong. int. pressions de terrains, B.A.N.F.F. 1977.

[4] D. F. Coates. Principe de la mécanique des roches monographie 874 .

Direction des mines, Ottawa - Canada.

[5] P. Morlier. Le fluage des roches.

Ann. I.T.B.T.P. $n^{\circ} 217$, Janvier 1966.

[6] J. Bonvallet. - M. Dejean. - R. Schwartzmann. Stabilité générale d'une carrière souterraine - application au cas des carrières d'Hordain (Nord).

Revue de I'Industrie Minérale, Août-Septembre 1978.

[7] P. Habib. Mesures des contraintes dans les piliers d'une carrière de craie.

Ann. I. T.B.T.P. Sup., Mai $1958, n^{\circ} 125$.

[8] M. Panet. Quelques remarques sur la stabilité des tunnels dans la craie.

Bull. Liaison P. et Ch., Spécial V 1973.

[9] J. L. Dessenne. - G. Comes. - P. Duffaut. P. Gérard. La craie au laboratoire et dans un tunnel profond.

VII ${ }^{\mathrm{e}}$ cong. int. mech. sols et trav. de fond. Thème IV, Mexico 1969. 
[10] J. L. Dessenne, - P. Duffaut. Les propriétés rhéologiques de la craie et leur influence sur le percement des galeries. La houille blanche. Mai 1970.

[11] G. Comes. - G. Akermann. La craie et la réfrigération des centrales électronucléaires situées sur le littoral.

Comité Franc. de Géol. de l'ing., Journées sur la craie, Rouen, 5-6 mai 1976.

[12] M. Rocha. - J. B. Lopes, - J.N. Da Silva. A new technique for applying the method J. N. the flat jack in the determination of stresses inside rock masses.

First congress of the international society for rock mechanics, Lisbon 1966.
[13] J. Bonvallet. - M. Dejean. Flat jack test and determination of mechanical caracteristics.

Field measurements in rock mechanics international symposi, Zurich, April 1977.

[14] S. Timoshenko. Théorie de l'élasticité, Dunod Paris.

[15] E. Tincelin. - P. Sinou. Effondrements brutaux et généralisés; coups de toit.

Revue de l'Industrie Minérale, Avril 1962.

[16] H. Labasse. Les pressions de terrains dans les carrières souterraines. Coups de toit et coups de charge.

Revue de I'Industrie Minérale, Août 1973. 
